2009-01-01

\title{
A Mobile ECG Monitoring System with Context Collection
}

Jin Peng Li

Technological University Dublin, jplidit@gmail.com

Damon Berry

Technological University Dublin, damon.berry@tudublin.ie

Richard Hayes

Technological University Dublin, richard.hayes@tudublin.ie

Follow this and additional works at: https://arrow.tudublin.ie/teapotcon

Part of the Biomedical Devices and Instrumentation Commons, Computer Engineering Commons, Databases and Information Systems Commons, and the Signal Processing Commons

\section{Recommended Citation}

$\mathrm{Li}$, J.P. and Berry, D. and Hayes, R. A mobile ECG monitoring system with context collection.4th European Conference of the International Federation for Medical and Biological Engineering,2009, pp.1222-125.

This Conference Paper is brought to you for free and open access by the tPOT: People Oriented Technology at ARROW@TU Dublin. It has been accepted for inclusion in Conference Papers by an authorized administrator of ARROW@TU Dublin. For more information, please contact arrow.admin@tudublin.ie, aisling.coyne@tudublin.ie, gerard.connolly@tudublin.ie. Funder: Council of Directors of IOTI, TSR Strand I 


\title{
A mobile ECG monitoring system with context collection
}

\author{
Li. J.P ${ }^{1}$, Berry $\mathrm{D}^{1}$ and Hayes $\mathrm{R}^{1}$ \\ ${ }^{1}$ Dublin Institute of Technology, Dublin, Ireland
}

\begin{abstract}
Preventative health management represents a shift from the traditional approach of reactive treatment-based healthcare towards a proactive wellness-management approach where patients are encouraged to stay healthy with expert support when they need it, at any location and any time. This work represents a step along the road towards proactive, preventative healthcare for cardiac patients. It seeks to develop a smart mobile ECG monitoring system that requests and records context information about what is happening around the subject when an arrhythmia event occurs. Context information about the subject's activities of daily living will, it is hoped, provide an enriched data set for clinicians and so improve clinical decision making. As a first step towards a mobile cardiac wellness guideline system, the authors present a system which can receive bio-signals that are wirelessly streamed across a body area network from Bluetooth enabled electrocardiographs. The system can store signals as they arrive while also responding to significant changes in Electrocardiogram activity. The authors have developed a prototype on a handheld computer that detects and responds to changes in the calculated heart rate as detected in an ECG signal. Although the general approach taken in this work could be applied to a wide range of bio-signals, the work focuses on ECG signals. The components of the system are, - A Bluetooth receiver, data collection and storage module - A real-time ECG beat detection algorithm. - An Event-Condition-Action (E-CA) rule base which decides when to request context information from the user. - A simple user interface which can request additional information from the user. A selection of real-time ECG detection algorithms were investigated for this work and one algorithm was tested in MATLAB and then implemented in Java. In order to collect ECG signals (and in principle any signals), the generalised data collection architecture has also been developed using Java and Bluetooth technology. An Event-Condition-Action (ECA) rule based expert system evaluates the changes in heart beat interval to decide when to interact with the user to request context information.
\end{abstract}

\section{Keywords - ECG, PDA, Holter and ECA.}

\section{INTRODUCTION}

Ambulatory ECG is mainly used to document and describe abnormal electrical activity in the heart. This can be random, spontaneous, sleep-related or caused by emotion or stress [1]. The capture and association of symptoms with disturbances in rhythm during daily activity, requires a record of the heart's electrical activity during that time [2], [3], [4]. This must be done continuously over time as a person goes about Activities of Daily Living (ADL) [5], [6]. In traditional holter recording, subjects often use diaries to record the activity that accompanies the biosignal recording. This work presents an approach where the biosignal recording system detects changes in the biosignal in realtime and prompts the user to provide additional context input about the activities of daily living (ADL).

\section{CONTEXT COLLECTION FOR MOBILE HEALTH MONITORING}

When a Holter-monitored subject is engaged in physical exertion such as ascending a long staircase, their heart rate will rise. If the subject's heart rate is monitored while they are carrying out this activity of daily living, and the context information about stairs climbing is not noted, the monitoring system is lacking information to interpret the raised heart rate so it is useful for an ECG system to be aware of the ADL context. As Jones [7] suggests "Timely processing and transmission of such multimedia clinical data in a distributed mobile environment requires smart strategies." Clearly the most effective smart strategies are the well informed ones and so context awareness becomes important. Some of the more sophisticated contemporary Holter monitoring systems can record and respond to ECG signals locally [8], [9], These systems in some cases incorporate an ECG analysis algorithm implemented in an embedded system or PDA that detects any abnormal heart activities and sends an alarm to the user and the remote monitoring centre.

Some contemporary systems [8],[9] also analyze the ECG locally and inform the patient about symptoms, thus allowing the patient to deal with the problem while issuing an alarm message to a control centre so that cardiac specialists can review the recorded abnormalities. These current systems [8], [10], [9] are 'not aware of' the daily activity context of the patient, only the ECG data. The expert system reported here will as much as possible facilitate better informed healthcare by collecting context information within a personal area network. 


\section{DESCRIPTION OF THE SYSTEM}

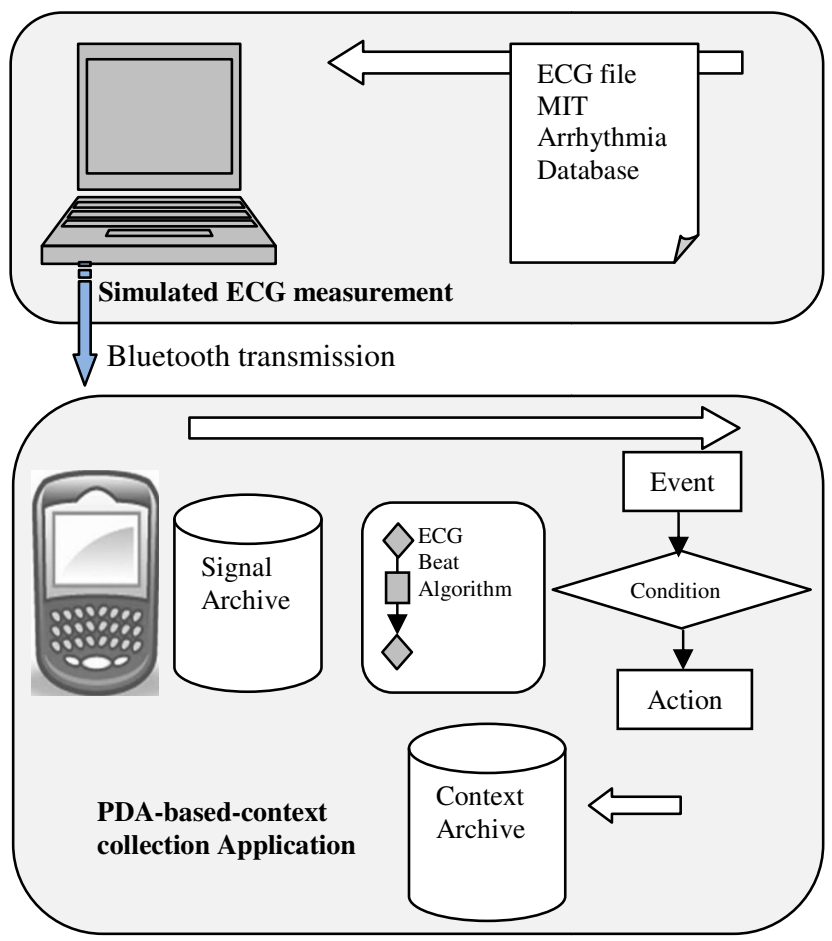

Figure 1: Overview of Intelligent Context Collection System for Activities of Daily Living

\section{A. Bluetooth Communications}

Bluetooth is an industrial specification that supports wireless communication within a short range [11] and Bluetooth has comparatively reliable performance which helps make it a suitable channel for medical communications [12]. Java Bluetooth applications are usually developed with the employment of the Standard Java Bluetooth API and the Serial Port Profile, a part of the Bluetooth stack that makes a Bluetooth channel behave as a simple wireless serial link. It emulates a serial cable to provide a simple-to-use wireless replacement for existing RS232 based serial communications applications. By providing interfaces to the communication ports using the Serial Port Profile, it is possible to build a Java application for short range communication usually at no extra cost.

\section{B. Beat Detection Algorithm}

In order to interact with the user in an event oriented fashion, it is necessary to raise events when significant features occur in the sampled ECG data stream. In this case the feature that is being monitored is a "beat". It is wellknown [13] that zero crossing beat detection methods are robust against noise as in the case of ambulatory ECG recording and are particularly useful for finite precision arithmetic. The Kohler zero crossing beat detection algorithm [13] was chosen for this work. The excellent performance of the Kohler algorithm was confirmed in other work [14] by a sensitivity of $99.70 \%$ (277 false negatives) and a positive "predictivity" of $99.57 \%$ (390 false positives) against the MIT-BIH arrhythmia database, where 135 MIT-BIH ECG records with 91006 real beats or nearly 24 hours of ECG recordings were tested under this algorithm.

The ECG signals can be processed by Kohler's algorithm in the following steps: 1) Filter the ECG signal first in order to attenuate the mean, the $\mathrm{P}$ - and $\mathrm{T}$-wave, and the high frequency noise; 2) Increase the signal quality by passing it through a band pass filter with a linear phase response; 3) a further increase in the quality of the band-passed ECG signal is achieved by the application of Equation 1 to the filtered digital signal.

$$
y(n)=\operatorname{sign}\left(x_{f}(n)\right) \cdot x_{f}^{2}(n)
$$

where $\mathrm{x}_{\mathrm{f}}(\mathrm{n})$ denotes the filtered ECG signal, $\operatorname{sign}()$ is a mathematical function which returns $1,0,-1$ depending on the inputted values and $y(n)$ is the result after this non-linear transformation of the filtered ECG signal. The signal $y(n)$ will be used later for the determination of the temporal location of the R-wave; 4) add a high-frequency sequence to the resulting waveform to facilitate the occurrence of zero crossing; 5) Detect Zero-Crossings: For this, Kohler recommends a term $\mathrm{d}$ that is calculated as follows:

$$
d(n)=\left|\frac{\operatorname{sign}\left[y^{\prime}(n)\right]-\operatorname{sign}\left[y^{\prime}(n-1)\right]}{2}\right|
$$

where the possible values for the sign [y' (n)] are $-1,0$, and 1 , therefore the possible values for the equation above are $0,0.5,1$. The feature signal is calculated as

$$
D(n)=\lambda_{D} \cdot D(n-1)+\left(1-\lambda_{D}\right) \cdot d(n)
$$

where $\lambda_{D}$ is a forget factor values from 0 to 1 . 6) The threshold for the feature signal is defined in Equation 4:

$$
\Theta(n)=\lambda_{\Theta} \cdot \Theta(n-1)+\left(1-\lambda_{\Theta}\right) \cdot D(n)
$$

where $\lambda_{\Theta}$ is a forgetting factor again which varies from 0 and 1 , which is a weighted sum that can be adjusted due to different situations. 


\section{E-C-A Rule parser}

The "beat" events generated by the Kohler algorithm described in the last section can now be processed in order to request user action and data input when this is required. Event-Condition-Action (E-C-A) rules are a mechanism first developed by Oracle [15] for use in active databases and they are a natural method for supporting reactive functionality [16], [17]. The important features of an E-C-A approach are its reactive and reasoning capabilities, the possibilities to express complex actions and events and declarative semantics [18], [19]. The form of E-C-A is On [Event] IF [Condition is TRUE] DO [Action], which means that when Event occurs, if Condition is verified, then execute Action. E-C-A systems receive inputs (mainly in the form of events) from the external environment and react by performing actions that change the stored information (internal actions) or influence the environment itself (external actions)[19]. Conditions can range from simple to compound logic statements.

The first stage of this parser is the Event that has a beat event listener. Once the beat event is generated, the beat event listener will notify the Event part to store this beat into an array of beat Events. Whenever there are two beat Events in the array, the time interval between neighbouring beats is calculated shown in Figure 2.

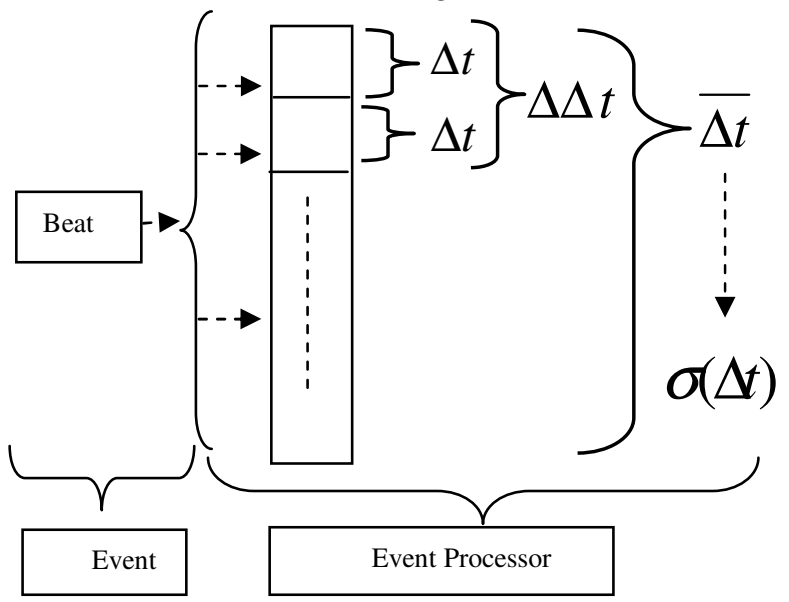

Figure 2 Event processing showing the calculation of heartbeat intervals and heart rate variability from beat event "queue"

The condition part also has a "listener" that listens to the calculated result from the Event part. A healthy adult should have a time interval between heart beats of around 800 milliseconds (75beats/min) [20]. So when the time interval has become much smaller or larger than 800 milliseconds, this condition part will place the calculated time interval into its personalized rule engine for evaluation to see if it is too low or too high shown in Figure 3. When evaluation is finished, the condition is alerted that the time interval is too low or too high. As a result it will notify the corresponding user interface which will ask the user what they are doing at that moment to gather the context information, as illustrated in Figure 3.

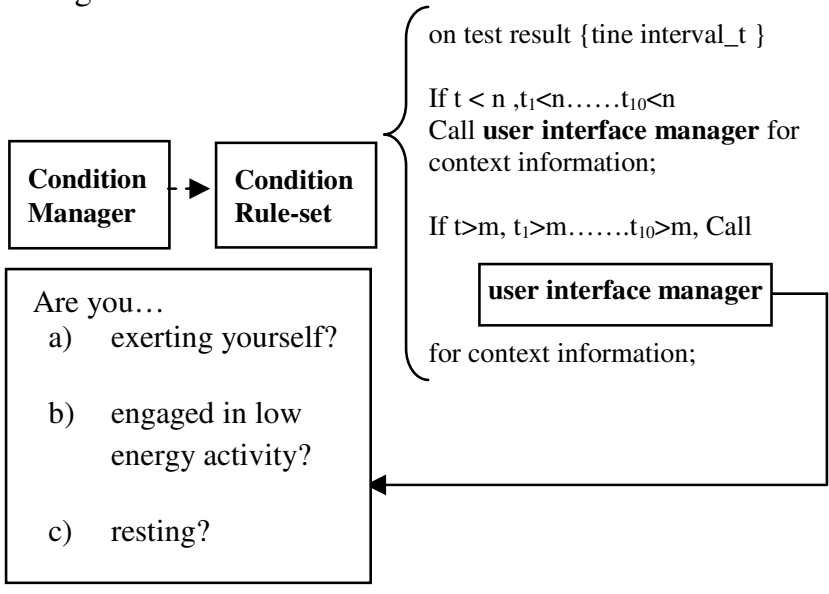

Figure 3 Condition evaluation and user interface

\section{RESULtS}

The context collection facility in this system is composed of three parts: data transportation, algorithm and E-C-A rule parser.

In agreement with the literature for Bluetooth data transportation, it was discovered that when the PDA is 30 meters away in line of sight from the simulator, it could no longer receive data from the Bluetooth channel.

The beat detection algorithm that was developed in Java, implements Kohler's zero-crossing algorithm [13] thus proving that it can work in real time and has low computational load and high detection accuracy.

The E-C-A rule parser has a memory efficient interface which uses a logger file. This file is used to record all the context information. When a suspicious heart beat interval is detected, the application asks the user to modify the logger file to input the current context information and also records the associated time information automatically.

\section{Conclusions}

This work presented a mobile intelligent context collection system and an accompanying healthcare scenario that can be generalized to represent a context information collection to support other mobile self-wellness monitoring systems such as respiration, glucose levels and EEG [21]. It 
can also in principle be applied to other time series signals in power signature analysis, and weather reporting.

Experiences of the authors during the project indicate that the contemporary mobile devices and wireless communications at time of writing still suffer from certain limitations which may restrict the ability to store, process and transmit large volumes of multimedia clinical data in real time. However, while mobile devices still have limited memory and processing power, and are especially restricted by their battery life, these capacities are increasing rapidly and it is the authors' firm view that the future is bright for mobile healthcare.

With the rapid advances in computational power and communications infrastructure PDA-like devices with low memory usage and integrated context information collection which are managed by the patient have the potential to be an important and powerful tool for the doctor to make a better decision for the user's health condition afterwards or on remote monitoring.

\section{REFERENCES}

1. Kowey P. R and Kocovic D. Z (1993) Ambulatory Electrocardiographic Recording, American Heart Association, ISSN: 0009-7322, Online 72514, pp 337-341

2. Kadish AH, Buxton AE, Kennedy HL (2001) ACC/AHA clinical competence statement on electrocardiography and ambulatory electrocardiography: a report of the ACC/AHA/ACP-ASIM task force on clinical competence, Circulation, 104:3169-3178

3. HL K., Podrid P. J (2001) Role of Holter monitoring and exercise testing for arrhythmia assessment and management, Cardiac Arrhythmia Philadelphia, Lippincott Williams \& Wilkins, pp 165-193

4. Ambulatory ECG at

http://www.americanheart.org/presenter.jhtml?identifier $=4425$

5. Chen G. and Kotz D (2000) A Survey of Context-Aware Mobile Computing Research, Computer Science Technical Report TR2000381, Department of Computer Science, Dartmouth College, Dartmouth, pp 1-3

6. Crawford M. H., Bernstein SJ, Deedwania PC, DiMarco JP, Ferrick KJ, Garson A Jr (1999) ACC/AHA guidelines for ambulatory electrocardiography, Journal of the American College of Cardiology, Volume 34, Issue 4, pp 1262-1347

7. Jones V.M, and Mei H, and Broens, T.H.F. and Widya, I.A. and Peuscher J (2007) Context Aware Body Area Networks for Telemedicine, 8th Pacific Rim Conference on Multimedia, Hong Kong, China, pp 590-599

8. Fensli R, Gunnarson E, Hejlesen O (2004) A Wireless ECG System for Continuous Event Recording and Communication to a Clinical Alarm Station, Agder University College, Faculty of Technology, Grimstad, Norway, Medinfo, Amsterdam: IOS Press, IMIA, pp 22082211

9. Rodríguez J, Goni A and Illarramendi (2005) A Real-Time Classification of ECGs on a PDA, IEEE Transactions on Volume 9, Issue 1, pp 23-34

10. Holter Devices Comparison http://www.medcompare.com/matrix/132/Holter-Monitor.html

11. Bluetooth at http://www.bluetooth.com/bluetooth/
12. Cronin A (2005) MPhil Thesis The Investigation of a Wireless Medical Homecare Data Logger, Dublin Institute of Technology, Department of Control Systems and Electrical Engineering pp 53-63

13. Kohler B.U, Orglmeister R (2003) "QRS Detection Using Zero Crossing Counts", Progress in Biomedical Research, 8 (3), pp 138145 .

14. Kohler B.U, Hennig C, and Orglmeister R (2002) "The Principles of Software QRS Detection", Engineering in Medicine and Biology Magazine, IEEE, Volume 21, Issue 1, pp 42-57, ISSN: 0739-5175

15. Oracle at www.oracle.com

16. Wu B and Dube K (2001) Applying Event-Condition-Action Mechanism in Healthcare: a Computerised Clinical Test-Ordering Protocol System (TOPS), Cooperative Database Systems for Advanced Applications, CODAS, IEEE INSPEC Accession Number: 7068993, ISBN: 0-7695-1128-7 pp 2 -9

17. Bailey J (2002) An Event-Condition-Action Language for XML, International World Wide Web Conference, Proceedings of the 11th international conference on World Wide Web, Honolulu, Hawaii, USA, pp $486-495$

18. Gatziu S (1998) Unbundling Active Functionality, ACM SIGMOD Record, Volume 27, Issue 1, pp 35-40

19. Zoumboulakis M (2004) Active Rules for Sensor Databases, ACM International Conference Proceeding Series, Volume 72, Proceeedings of the 1st international workshop on Data management for sensor networks: in conjunction with VLDB, Toronto, Canada, Session: Programming languages and architectures, pp 98-103

20. Rickards A. F (1996) An Implantable Intracardiac Accelerometer for Monitoring Myocardial Contractility, Pacing and Clinical Electrophysiology, Volume 19, Issue 12, pp 2066-2071

21. Papadelis C, Kourtidou-Papadeli C, Bamidis P. D, Chouvarda I (2006) "Indicators of Sleepiness in an ambulatory EEG study of night driving, Proceedings of the 28th IEEE EMBS Annual International Conference, New York City, USA

22. Podrid P. J, Kowey P (2001) Cardiac Arrhythmia Philadelphia, Lippincott Williams \& Wilkins, pp165-193 